Research Article

\title{
Stimulation of Codonopsis pilosula Polysaccharide on Bifidobacterium of Human Gut Bacteria In Vitro
}

\author{
Jiankuan Li $\mathbb{D}^{1},{ }^{1}$ Lina Dong, ${ }^{2}$ Yue Liu, ${ }^{3}$ and Jianping Gao ${ }^{1}$ \\ ${ }^{1}$ School of Pharmaceutical Science, Shanxi Medical University, Taiyuan 030001, China \\ ${ }^{2}$ Central Laboratory, Shanxi Provincial People's Hospital, Affiliate of Shanxi Medical University, Taiyuan 030012, China \\ ${ }^{3}$ China Institutes of Biomedical Sciences, Shanxi University, Taiyuan 030006, China
}

Correspondence should be addressed to Jiankuan Li; lijiankuan2004@163.com

Received 10 April 2020; Revised 10 September 2020; Accepted 20 March 2021; Published 30 March 2021

Academic Editor: Krishnadas Nandakumar

Copyright ( $\odot 2021$ Jiankuan Li et al. This is an open access article distributed under the Creative Commons Attribution License, which permits unrestricted use, distribution, and reproduction in any medium, provided the original work is properly cited.

Objective. To evaluate the prebiotic effects of Codonopsis pilosula polysaccharide (CPP) on human gut bacteria in vitro. Methods. Codonopsis Radix was extracted with water at $100^{\circ} \mathrm{C}$, and the extract was precipitated by $80 \%$ ethanol to obtain CPP. Human fresh fecal samples were collected from three healthy adults and used to ferment CPP. The fermented samples were collected to be analyzed by $16 \mathrm{~S}$ rRNA sequencing. Results. The results showed that CPP exhibited significantly the stimulation on the growth of genus Bifidobacterium of human gut bacteria $(\mathrm{Padj}<0.05)$. Although CPP also exhibited regulative trends on the genera including Acidaminococcus, Bilophila, Dorea, and Eggerthella, no significant differences were observed (Padj $>0.05$ ), which was likely associated with the limited samples $(n=3)$. Conclusion. CPP has the potential to stimulate the growth of Bifidobacterium of the human gut bacteria and to be benefit to human health.

\section{Introduction}

Codonopsis Radix (commonly named Dangshen in Chinese), derived from the roots of Codonopsis pilosula (Franch.) Nannf., Codonopsis pilosula (Franch.) Nannf. Var. modesta (Nannf.) L. T. Shen, and Codonopsis tangshen Oliv., has been usually used in many traditional Chinese medicine preparations for tonifying the spleen and lung in China [1]. Polysaccharides are traditionally considered to be significant active components responsible for the tonifying function of Codonopsis Radix. Recently, increasing evidences indicated that Codonopsis pilosula polysaccharides exhibited a variety of biological activities. Zhang et al. reported that Codonopsis pilosula polysaccharides attenuated tau hyperphosphorylation and cognitive impairments in adeno-associated virus serotype-induced expression of human full length tau in C57/BL6 mice [2]. Fu et al. reported that Codonopsis pilosula polysaccharides exhibited the protection against cyclophosphamide-induced immunosuppression in mice [3]. Pectin-type polysaccharides from Codonopsis pilosula were reported to exhibit immune-stimulation activity in RAW264.7 macrophage and the selenizing Codonopsis pilosula polysaccharides protected RAW264.7cells from hydrogen peroxide-induced injury $[4,5]$.

The human gastrointestinal tract is enriched with a complex population of microorganisms, which exert a significant influence on the host health and disease [6]. Previously, inulin-type fructans were obtained from Codonopsis pilosula and showed prebiotic activity by stimulating the growth of Lactobacillus and Bifidobacterium in vitro [7, 8]. Jing et al. reported that Codonopsis pilosula polysaccharides exhibited prebiotic-like effects in dextran sulfate sodiuminduced colitis mice by stimulating the growth of Bifidobacterium, Lactobacillus, and Akkermansia probiotics [9]. The present study intends to investigate the modulation of $\mathrm{CPP}$ on human gut bacteria by in vitro fermentation.

\section{Methods}

2.1. Plant Material. Roots of Codonopsis pilosula (Franch.) Nannf. were collected from Pingshun county in Shanxi province, China, and were identified by Professor Jianping 
Gao (School of Pharmaceutical Science, Shanxi Medical University) to be Codonopsis Radix. A voucher specimen (2018-09-MCM) was deposited at Herbarium, School of Pharmaceutical Science, Shanxi Medical University.

2.2. Extraction of Polysaccharide. Dried roots of Codonopsis pilosula were crushed and extracted two times with water $(10: 1, \mathrm{v} / \mathrm{w})$ at $100^{\circ} \mathrm{C}$ for $2 \mathrm{~h}$. The combined extract was concentrated to $1 / 4$ volume under vacuum at $60^{\circ} \mathrm{C}$ and then was precipitated with $80 \%$ ethanol and centrifuged for $15 \mathrm{~min}$ at $4000 \mathrm{rpm} / \mathrm{min}$ to obtain CPP. Proteins were removed from CPP using the Sevag reagent (chloroform/ butanol, $v / v=4: 1)$.

2.3. Fecal Sample Collection and Fermentation. Fresh fecal samples $(10 \mathrm{~g})$ were collected from three healthy adults and promptly suspended in pre-reduced phosphate-buffered saline (PBS). The samples were then added in $50 \mathrm{~mL}$ centrifuge tube pre-placed sterile filter bag and filtered. CPP was added in $100 \mathrm{~mL}$ filtered liquid at a ratio of $1: 100(\mathrm{~g} / \mathrm{mL})$ and fermented in anaerobic condition for 0,8 , and $24 \mathrm{~h}$. After fermentation, $50 \mathrm{~mL}$ fermented liquid was filtered and the precipitate was added with $2 \mathrm{~mL}$ pre-reduced glycerinPBS $(1: 1)$ and then centrifuged for $10 \mathrm{~min}$ at $4,000 \mathrm{rpm} / \mathrm{min}$; the precipitate immediately was kept at $-80^{\circ} \mathrm{C}$ for $16 \mathrm{~S}$ rRNA gene sequencing analysis (DeepBiomedical Co., Ltd, Jinan, China).

2.4. $16 S$ rRNA Sequencing. The DNA was extracted from $100 \mathrm{mg}$ samples using DNeasy Power Soil Kit (Cat. 47014, Qiagen, Germany). The DNA concentration was measured with Nanodrop (Thermo scientific). The DNA was diluted to proper concentration for further 16S rRNA gene fragments (V3-V4) amplification. The polymerase used for $16 \mathrm{~S}$ rRNA gene amplification was Phanta Max Master Mix (Vazyme Biotech Co., Ltd. Nanjing, China) following the manufacturer's procedure. The sequencing was implemented at Novogene Co. (Beijing, China) with a $2 \times 250$-bp paired-end sequencing strategy.

The V3-V4 regions of microbial 16S rDNA genes were amplified with primers of 341F ( $5^{\prime}$-CCTAYGGGRBGCASCAG- $3^{\prime}$ ) and 806R (5'GGACTACNNGGGTATCTAA $\left.\mathrm{T}-3^{\prime}\right)$. The $25 \mu \mathrm{l}$ PCR amplification mixture contained $25 \mathrm{ng}$ DNA, $1 \mu \mathrm{l}$ each primer $(10 \mu \mathrm{M}), 0.5 \mu \mathrm{l}$ dNTP $(2.5 \mathrm{mM})$, $12.5 \mu \mathrm{l}$ Vazyme Phata max buffer, and $0.5 \mu \mathrm{l}$ vazyme polymerase (Vazyme Biotech). The PCR was performed with an initial denaturation ( 5 minutes at $95^{\circ} \mathrm{C}$ ), followed by 27 cycles of 15 seconds at $95^{\circ} \mathrm{C}, 15$ seconds at $55^{\circ} \mathrm{C}$, and 30 seconds at $72^{\circ} \mathrm{C}$, and final with one cycle of $5 \mathrm{~min}$ at $72^{\circ} \mathrm{C}$. The PCR products were purified with the KAPA Pure Beads (Roche) according to the manufacturer's instructions and further sequenced with an Illumina NovaSeq 6000 system (Illumina). The sequences were then clustered into OTUs (operational taxonomic units) at 99\% identity thresholds using MOTHUR. Representative sequences of OTUs were selected and compared with ribosomal RNA database to obtain species annotation information. According to species annotation information, OTUs were filtered to obtained valid OTUs.

2.5. Statistical Analysis. Basing on the absolute abundance of valid OTUs and annotation information, the genus taxonomic level was obtained for differential abundance analysis with DEseq2 (version 1.26.0), Padj< 0.05 was considered to be significant difference.

\section{Results and Discussion}

3.1. Stimulation of CPP on the Growth of Bifidobacterium and Acidaminococcus. As shown in Figure 1, the genus Bifidobacterium growth (Figure 1(a)) was observed to be significantly stimulated by CPP after fermentation for 8 and $24 \mathrm{~h}$. The previous study by Jing et al. showed that Codonopsis pilosula polysaccharides exhibited the stimulation on the growth of Bifidobacterium, Lactobacillus, and Akkermansia probiotics in dextran sulfate sodium-induced colitis mice [9]. The present study showed that only Bifidobacterium of human gut bacteria was stimulated by CPP during $24 \mathrm{~h}$ fermentation. It was well known that Bifidobacterium was important probiotics. So, it was indicated that CPP was potential prebiotics by stimulating the growth of Bifidobacterium both in vivo and in vitro, which could be explained by the fact that Codonopsis Radix was rich in insulin-type fructans [8].

Meanwhile, CCP also exhibited a stimulatory trend on the growth of Acidaminococcus (Figure 1(b)) but no significant difference was observed (Padj>0.05). Acidaminococcus was first reported by Rogosa to be using amino acid as the sole energy source for growth [10]. The effects of Acidaminococcus on human health were unclear. Previously, Qi et al. found that the composition of Haemophilus, Lachnospira, Dialister, and Acidaminococcus was decreased significantly in T1DM Chinese children compared to controls [11]. Li et al. found that the relative abundances of Acidaminococcus, Succinivibrio, and Citrobacter were reduced obviously in canines with high fat diet-induced obesity, while green tea polyphenols could increase the abundances of Acidaminococcus, Succinivibrio, and Citrobacter [12]. These studies indicated in some ways that Acidaminococcus could be associated negatively with metabolic diseases such as obesity and diabetes mellitus.

3.2. Inhibition of CPP on the Growth of Bilophila, Dorea, and Eggerthella. As shown in Figure 2, CPP exhibited inhibitory trends on the growth of three genera including Bilophila (Figure 2(a)), Dorea (Figure 2(b)), and Eggerthella (Figure 2(c)) after fermentation for 8 and $24 \mathrm{~h}$, but no significant differences were observed (Padj>0.05). In the control group, Bilophila, Dorea and Eggerthella exhibited a natural growth tendency, while they exhibited almost no growth tendency in CPP group. Natividad et al. reported that Bilophila wadsworthia with high-fat diet promoted higher inflammation, intestinal barrier dysfunction, and bile acid dysmetabolism, leading to higher glucose dysmetabolism 


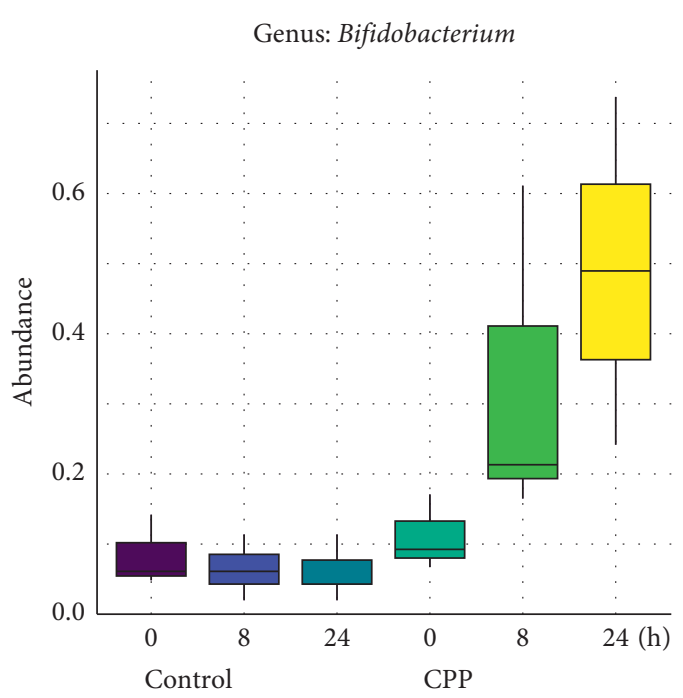

(a)

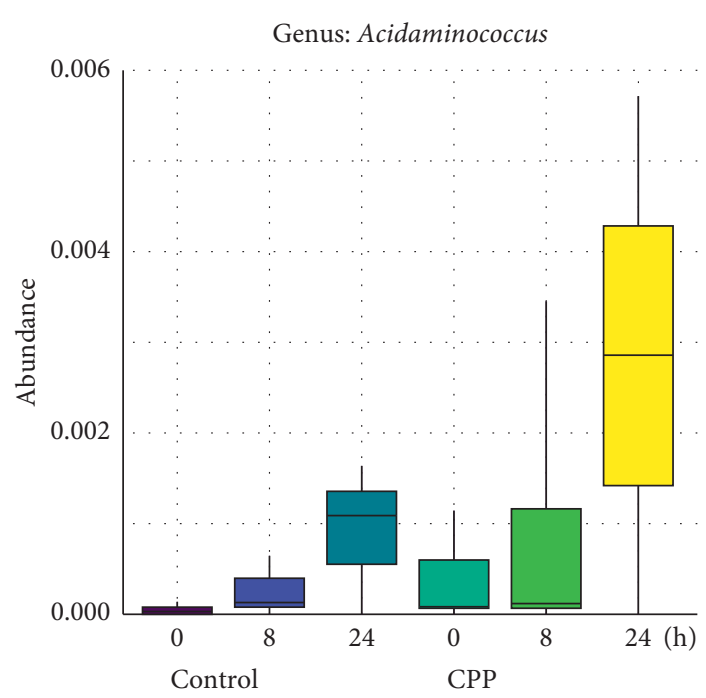

(b)

Figure 1: Stimulation of CPP on Bifidobacterium (a); control 24 versus CPP 24, Padj< 0.05) and Acidaminococcus (b).

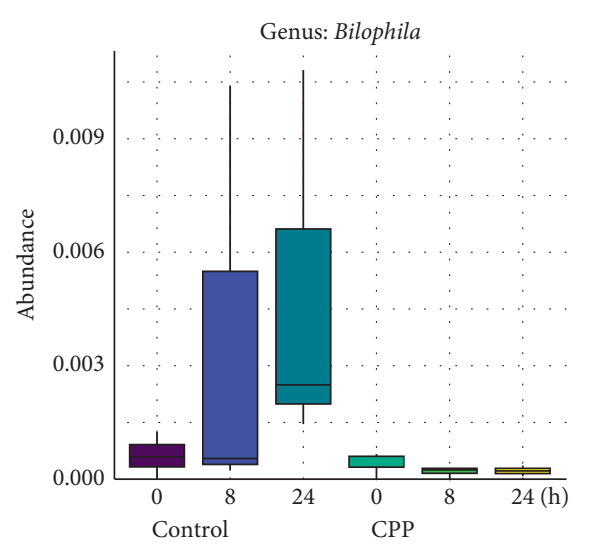

(a)

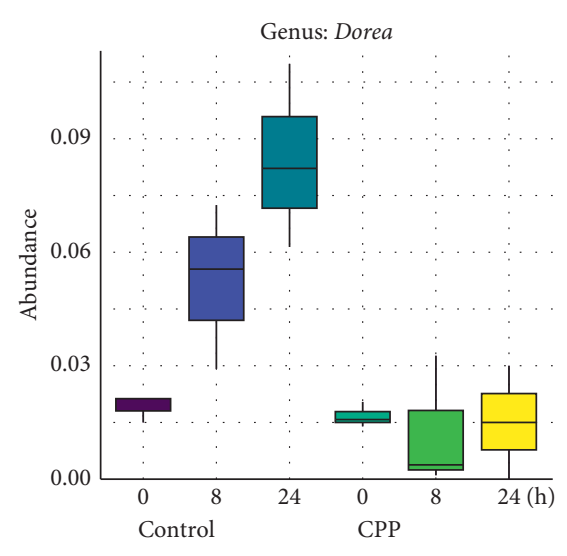

(b)

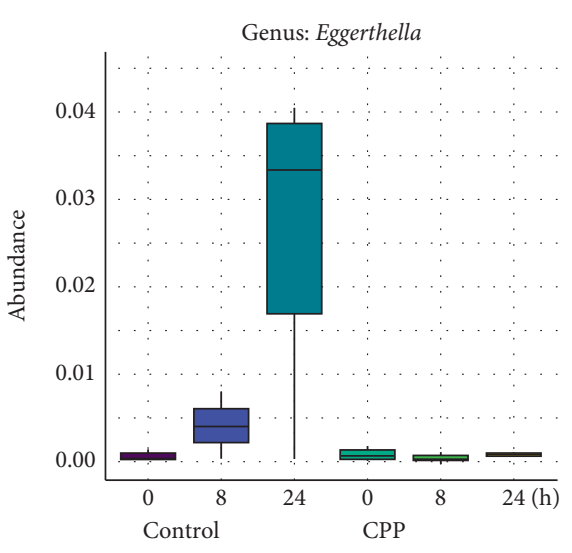

(c)

FIgure 2: Inhibition of CPP on Bilophila (a), Dorea, (b) and Eggerthella (c).

and hepatic steatosis in mice [13], while the probiotic Lactobacillus rhamnosus CNCM I-3690 reduced Bilophila wadsworthia-induced immune and metabolic impairment. Bilophila wadsworthia was also reported to be more abundant in the colonic microbiome of colorectal cancer cases compared to healthy controls [14]. These data indicated that the genus Bilophila could likely be associated positively with inflammation.

Fang et al. compared the intestinal microbial diversity of healthy people and amyotrophic lateral sclerosis patients, which showed that the genus Dorea (harmful microorganisms) was significantly increased in amyotrophic lateral sclerosis patients [15]. The study by Chiu et al. showed that genus Dorea exhibited a significant correlation with allergic rhinitis [16]. Nitsan et al. found that the luminal proportions of Faecalibacterium and Dorea were significantly higher in fecal samples of irritable bowel syndrome (IBS) patient than those in fecal samples of healthy controls [17].

Sato et al. reported that the higher proportions of genera Parabacteroides and Eggerthella were associated with small newborn head circumference or weight in males [18]. Eggerthella lenta, a major cause of bacteremia, was reported to be a significant human pathogen that is often associated with serious gastrointestinal tract (GIT) pathology $[19,20]$.

CPP exhibited regulatory trends on the genera including Acidaminococcus, Bilophila, Dorea, and Eggerthella, but no significant differences were observed, which was likely associated with the limited samples in the study. So, it was suggested to increase samples in future studies to further investigate the modulation of CPP on the genera Acidaminococcus, Bilophila, Dorea, and Eggerthella. 


\section{Conclusion}

CPP exhibits the potential to stimulate the growth of Bifidobacterium of the human gut bacteria and likely to be benefit to human health.

\section{Data Availability}

The data used to support the findings of this study are available from the corresponding author upon request.

\section{Conflicts of Interest}

The authors declare no conflicts of interest.

\section{Acknowledgments}

This study was funded by the National Key R\&D Program of China (Nos. 2019YFC1710800 and 2018YFC1706300).

\section{References}

[1] S.-M. Gao, J.-S. Liu, M. Wang et al., "Traditional uses, phytochemistry, pharmacology and toxicology of Codonopsis: a review," Journal of Ethnopharmacology, vol. 219, pp. 50-70, 2018.

[2] Q. Zhang, Y. Xia, H. Luo et al., "Codonopsis pilosula polysaccharide attenuates tau hyperphosphorylation and cognitive impairments in hTau infected mice," Frontiers in Molecular Neuroscience, vol. 11, p. 437, 2018.

[3] Y.-P. Fu, B. Feng, Z.-K. Zhu et al., "The polysaccharides from Codonopsis pilosula modulates the immunity and intestinal microbiota of cyclophosphamide-treated immunosuppressed mice," Molecules, vol. 23, no. 7, p. 1801, 2018.

[4] Q.-L. Sun, Y.-X. Li, Y.-S. Cui, S.-L. Jiang, C.-X. Dong, and J. Du, "Structural characterization of three polysaccharides from the roots of Codonopsis pilosula and their immunomodulatory effects on RAW264.7 macrophages," International Journal of Biological Macromolecules, vol. 130, pp. 556-563, 2019.

[5] T. Qin, Z. Ren, X. Liu et al., "Study of the selenizing Codonopsis pilosula polysaccharides protects RAW264.7 cells from hydrogen peroxide-induced injury," International Journal of Biological Macromolecules, vol. 125, pp. 534-543, 2019.

[6] E. Thursby and N. Juge, "Introduction to the human gut microbiota," Biochemical Journal, vol. 474, no. 11, pp. 1823-1836, 2017.

[7] Y.-P. Fu, L.-X. Li, B.-Z. Zhang et al., "Characterization and prebiotic activity in vitro of inulin-type fructan from Codonopsis pilosula roots," Carbohydrate Polymers, vol. 193, pp. 212-220, 2018.

[8] J. K. Li, X. Zhang, L. Y. Cao et al., "Three inulin-type fructans from Codonopsis pilosula (franch.) Nannf. Roots and their prebiotic activity on Bifidobacterium longum," Molecules, vol. 23, pp. 1-11, 2018.

[9] Y. Jing, A. Li, Z. Liu et al., "Absorption of Codonopsis pilosula saponins by coexisting polysaccharides alleviates gut microbial dysbiosis with dextran sulfate sodium-induced colitis in model mice," BioMed Research International, vol. 2018, Article ID 1781036, 18 pages, 2018.

[10] M. Rogosa, "Acidaminococcus gen. n., Acidaminococcus fermentans sp. n., anaerobic gram-negative diplococci using amino acids as the sole energy source for growth," Journal of Bacteriology, vol. 98, no. 2, pp. 756-766, 1969.

[11] C.-J. Qi, Q. Zhang, M. Yu et al., "Imbalance of fecal microbiota at newly diagnosed type 1 diabetes in Chinese children," Chinese Medical Journal, vol. 129, no. 11, pp. 1298-1304, 2016.

[12] Y. Li, S. U. Rahman, Y. Huang et al., "Green tea polyphenols decrease weight gain, ameliorate alteration of gut microbiota, and mitigate intestinal inflammation in Canines with highfat-diet-induced obesity," The Journal of Nutritional Biochemistry, vol. 78, p. 108324, 2020.

[13] J. M. Natividad, B. Lamas, H. P. Pham et al., "Bilophila wadsworthia aggravates high fat diet induced metabolic dysfunctions in mice," Nature Communications, vol. 9, no. 1, p. 2802, 2018.

[14] C. Yazici, P. G. Wolf, T. P. Carroll et al., "Bilophila wadsworthia is more abundant in the colonic microbiome of colorectal cancer cases compared to healthy controls," AGA Abstracts, vol. 100, p. 511, 2017.

[15] X. Fang, X. Wang, S. Yang et al., "Evaluation of the microbial diversity in amyotrophic lateral sclerosis using highthroughput sequencing," Frontiers in Microbiology, vol. 7, p. 1479, 2016.

[16] C.-Y. Chiu, Y.-L. Chan, M.-H. Tsai, C.-J. Wang, M.-H. Chiang, and C.-C. Chiu, "Gut microbial dysbiosis is associated with allergen-specific IgE responses in young children with airway allergies," World Allergy Organization Journal, vol. 12, no. 3, p. 100021, 2019.

[17] M. Nitsan, R. Yehuda, K. David et al., "Fecal and mucosaassociated intestinal microbiota in patients with diarrheapredominant irritable bowel syndrome," Digestive Diseases and Sciences, vol. 63, pp. 1890-1899, 2018.

[18] Y. Sato, K. Sakurai, H. Tanabe et al., "Maternal gut microbiota is associated with newborn anthropometrics in a sex-specific manner," Journal of Developmental Origins of Health and Disease, vol. 10, no. 6, pp. 659-666, 2019.

[19] B. J. Gardiner, A. Y. Tai, D. Kotsanas et al., "Clinical and microbiological characteristics of Eggerthella lenta bacteremia," Journal of Clinical Microbiology, vol. 53, no. 2, pp. 626-635, 2015.

[20] U.-T. Alejandra, G. Mark R, G. Thomas et al., "Eggerthella lenta bloodstream infections are associated with increased mortality following empiric Piperacillin-Tazobactam (TZP) monotherapy: a population-based cohort study," Clinical Infectious Diseases, vol. 67, pp. 221-228, 2018. 